\title{
Epidemiology, Major Outcomes, Risk Factors, Prevention and Management of Chronic Kidney Disease in China
}

\author{
Yuansheng Xie Xiangmei Chen \\ Department of Nephrology, Chinese General Hospital of PLA, Beijing, China
}

\section{Key Words}

Chronic kidney disease $\cdot \lg$ A nephropathy · Lupus nephritis • Prevalence, China renal biopsy in China. Chinese multicenter clinical trials showed that mycophenolate mofetil or leflunomide combined with steroids was effective as induction therapy for proliferative LN.

\begin{abstract}
Chronic kidney disease (CKD) is common in China. In residents older than 40 years in Beijing, China, $11.3 \%$ of subjects had at least one indicator of kidney damage. The primary cause of chronic renal failure in China was glomerulonephritis, which was followed by diabetic nephropathy and hypertensive nephrosclerosis. Renal failure, cardiovascular disease and infection were important complications. IgA nephropathy $(\lg A N)$ is the most common CKD in China. The prevalence of hypertension, intrarenal artery lesions and tubulointerstitial lesions in patients with $\lg \mathrm{AN}$ at the time of renal biopsy was approximately 40, 55 and 85\%, respectively. The genetic variation in Megsin confers susceptibility to IgAN in Chinese. The patients with SL/LL genotypes of the MUC20 gene, the 38AA genotype of uteroglobin and DD genotype of the angiotensin-converting enzyme gene had a higher risk of progression. Chinese prospective clinical trials showed that benazepril (BZ) conferred substantial renal benefits in patients with advanced renal insufficiency. The combined therapy with urokinase and $B Z$ was more effective than with $B Z$ alone in reducing proteinuria and protecting renal function in Chinese patients with severe IgAN. Lupus nephritis (LN) is a common form of secondary renal disease diagnosed by
\end{abstract}

\section{KARGER}

Fax +41613061234

E-Mail karger@karger.ch

www.karger.com
(C) 2007 S. Karger AG, Basel

0250-8095/08/0281-0001\$24.50/0

Accessible online at:

www.karger.com/ajn
Chronic kidney disease (CKD) is a worldwide public health problem with an increasing incidence and prevalence, poor outcome and high costs [1]. The causes of CKD are multiple and there are prominent differences regarding race and the geographical distribution of the disease. Thus, the strategies for prevention and treatment of CKD may differ among countries. This review presents the epidemiology, major outcomes and risk factors of CKD in China. We specifically point out that the equation of the Modification of Diet in Renal Disease (MDRD) is necessary for diagnosis of CKD in Chinese patients. Strategies for the prevention and management of CKD in China were also discussed. CKD in China shares many characteristics with that in developed countries, but at the same time it also has its own unique features.

IgA nephropathy (IgAN) was the most common CKD, and primary glomerular disease was the primary cause of chronic renal failure (CRF) in China. In recent years, however, the prevalence of diabetic nephropathy seems to increase rapidly.
Prof. Xiangmei Chen

Department of Nephrology

Chinese General Hospital of PLA, Fuxing Road 28

Beijing 100853 (China)

Tel. +86106693 5462, Fax +86 106813 0297, E-Mail xmchen301@126.com 


\section{Definition of CKD and Glomerular Filtration Rate in Chinese}

In February 2002, the Kidney Disease Outcomes Quality Initiative (K/DOQI) of the National Kidney Foundation published 15 clinical practice guidelines on CKD [2]. The Chinese Society of Nephrology (CSN) has accepted the definition and five-stage classification system of CKD and the major recommendations on early detection in adults. CKD is defined as either kidney damage or a glomerular filtration rate (GFR) $<60 \mathrm{ml} /$ min per $1.73 \mathrm{~m}^{2}$ for 3 or more months. Kidney damage is defined as pathologic abnormalities or markers of damage, including abnormalities in blood or urine tests or imaging studies. Persistent proteinuria is the principal marker of kidney damage [3]. GFR can be estimated from calibrated serum creatinine and estimating equations, e.g. the MDRD Study equation or the Cockcroft-Gault formula.

The MDRD formula is recommended by European and American guidelines for estimating GFR, but has not been fully validated in different populations and at different stages of CKD [4]. Zuo et al. [5] reported that in a Chinese population with CKD, the abbreviated MDRD equation overestimated GFR in patients with CKD stages 4-5 and underestimated GFR in those with CKD stage 1, suggesting that a modification of these equations is necessary for their application in Chinese patients with CKD. Ma et al. [6] modified MDRD equations based on data from Chinese CKD patients and compared the diagnostic performance of the modified MDRD equations with that of the original MDRD equations across CKD stages in a multicenter, cross-sectional study of GFR estimation from plasma creatinine $\left(\mathrm{P}_{\mathrm{cr}}\right)$, demographic data and clinical characteristics. The original MDRD equations were modified by two methods: first, by adding a racial factor for Chinese in the original MDRD equations, and, second, by applying multiple linear regression to the training sample and modifying the coefficient that is associated with each variable in the original MDRD equations and then validating in the testing sample and comparing it with the original MDRD equations. All modified MDRD equations showed significant performance improvement in bias, precision and accuracy compared with the original MDRD equations. By adding racial coefficients, the final equations were re-expressed as follows [6]:

The MDRD equation 7 to estimate GFR $(\mathrm{ml} / \mathrm{min}$ per $\left.1.73 \mathrm{~m}^{2}\right)=170 \times \mathrm{P}_{\mathrm{cr}}{ }^{-0.999} \times \mathrm{age}^{-0.176} \times \mathrm{BUN}^{-0.170} \times$ albumin $^{0.318} \times 0.762$ (if female) $\times 1.211$ (if Chinese).
Abbreviated MDRD equation to estimate GFR (ml/ min per $1.73 \mathrm{~m}^{2}$ ) $=186 \times \mathrm{P}_{\mathrm{cr}}{ }^{-1.154} \times$ age $^{-0.203} \times 0.742$ (if female) $\times 1.233$ (if Chinese), where $\mathrm{P}_{\mathrm{cr}}$ is in $\mathrm{mg} / \mathrm{dl}$, BUN is in $\mathrm{mg} / \mathrm{dl}$, albumin is in $\mathrm{g} / \mathrm{dl}$, and age is in years. However, further verification of these equations is necessary in larger Chinese study cohorts with different nation, age and health status.

\section{Epidemiology of CKD in China}

Worldwide, increasing numbers of patients are affected by CKD. In the USA, data derived from the Third National Health and Nutrition Examination Survey (NHANES III) have implied that up to $11 \%$ of the general adult population aged 20 years and older could have some degree of CKD [7]. Of 11,247 noninstitutionalized Australians aged 25 year or over, approximately $16 \%$ have at least one indicator of kidney damage, either proteinuria, hematuria and/or reduced GFR, indicating the presence of kidney damage [8]. There are no data regarding incidence and prevalence of nationwide CKD in China. In Chinese adults aged 35-74 years, the overall prevalence of CKD with GFR $<60 \mathrm{ml} / \mathrm{min} / 1.73 \mathrm{~m}^{2}$ was $2.5 \%$ [9]. In the residents ( $>40$ years old) in one district of Beijing, China, albuminuria was detected in $6.2 \%$ of subjects; reduced renal function was found in $5.2 \%$ of subjects; hematuria in $0.8 \%$ and non-infective pyuria in $0.09 \%$. Approximately $11.3 \%$ of subjects had at least one indicator of kidney damage [10]. In the general adult population ( $>20$ years) of Guangzhou city (Southern China), the prevalence of albuminuria was $6.16 \%$, hematuria $6.5 \%$ and reduced estimated GFR 1.64\%. Approximately $10.1 \%$ of subjects had at least one indicator of kidney damage [11]. In the residents $>18$ years in a village of Zhejiang Province, China, the prevalence of albuminuria was $10.4 \%$, hematuria $1.4 \%$ and reduced estimated GFR 3.0\%. Approximately $13.5 \%$ of subjects had at least one indicator of kidney damage [12] (table 1).

Renal biopsy was routinely performed in China in the last 20 years. Analysis of renal biopsy data of 13,519 Chinese patients showed that primary glomerulonephritis (GN) remained the most important and prevalent renal disease in China. The ratio of primary to secondary GN was 2.75:1. However, it is progressively declining in the past decades with an increment in the incidence of diabetic nephropathy and nephrosclerosis. IgAN constituted $45.3 \%$ of the primary GN. The most prevalent etiology of secondary GN was systemic lupus erythematosus followed by Henoch-Schönlein purpura and diabetic ne- 
phropathy [13]. However, a lot of patients with clinical diabetic nephropathy had not been biopsied. In fact, the prevalence of diabetic nephropathy increases rapidly with increasing prevalence of diabetes mellitus. In the past 20 years, due to a change in the diagnosis of renal disease proven by renal biopsy, the number of patients with acute renal failure (ARF), secondary renal disease and renal tubulointerstitial disease increased in the elderly in China [14].

The worldwide rise in the number of patients with CKD is reflected in the increasing number of patients with end-stage renal disease (ESRD) treated by renal replacement therapy, dialysis or transplantation [15]. In Europe, the annual average incidence of ESRD is 135 new patients per million population and the prevalence of ESRD is 700 patients per million population [16]. In the USA, the annual incidence of ESRD has reached 336 new patients per million population and the prevalence of ESRD has reached 1,403 patients per million population [17]. In a study on $607 \mathrm{CRF}$ cases with serum creatinine persistently $>3.0 \mathrm{mg} / \mathrm{dl}$ conducted in Nanjing, China, from 1979 to 2002 on biopsy samples, primary glomerular diseases were identified as the most frequent cause of CRF, e.g. IgAN and focal segmental glomerulosclerosis [13]. From January 1 to December 31, 1999, registration of dialysis and transplantation in whole China, except Hong Kong, Macao and Taiwan, was performed by the Dialysis and Transplantation Registration Group, CSN, using a questionnaire. In China, the first cause of CRF was GN followed by diabetic nephropathy and hypertensive nephrosclerosis [18]. In Shanghai, the annual incidence rate of dialysis was 155 per million population and point prevalence (December 31,1999 ) was 214 per million population [19]. These data showed that the annual incidence rate of dialysis in Shanghai, China, was similar to the annual average incidence of ESRD in Europe. However, prevalence of dialysis was much lower in Shanghai than in Europe. The financial problem may contribute to the difference.

\section{The Major Outcomes of CKD in China}

The major outcomes of CKD include progression to kidney failure, complications of decreased kidney function and cardiovascular disease (CVD). The occurrence of ARF in CKD (A/C) is an important complication of CKD. Drug-induced acute renal interstitial/tubulointerstitial disease, prerenal ARF and flare-up of lupus nephritis (LN) were the most common causes of A/C in China.
Table 1. Prevalence of CKD in different Chinese areas

\begin{tabular}{lcll}
\hline Area & Subjects & $\begin{array}{l}\text { Prevalence } \\
\text { of CKD, \% }\end{array}$ & $\begin{array}{l}\text { Reference } \\
\text { No. }\end{array}$ \\
\hline Beijing city & 2,353 & 11.3 & 10 \\
Guangzhou city & 2,213 & 10.1 & 11 \\
Zhejian village & 795 & 13.5 & 12 \\
\hline
\end{tabular}

More than one third of $\mathrm{A} / \mathrm{C}$ was associated with drugs, e.g. antibiotics and analgesics, which occurred more commonly in older patients [20].

CVD is one of the most serious and important complications of CKD. The risk of CVD is significantly increased even in patients with minor CKD [21]. A multicenter Chinese cohort study including 1,239 CKD patients from seven major medical centers showed that the most prevalent form of CVD was left ventricular hypertrophy, accounting for $58.5 \%$. The prevalence of congestive heart failure, coronary artery disease and cerebrovascular accidents was 27.7, 16.5 and 5.6\%, respectively. In the cohort with minor renal dysfunction (stage 2-3), coronary artery disease and cerebrovascular accidents were more prevalent than in the general population in the same regions. The prevalence of coronary artery disease, left ventricular hypertrophy and congestive heart failure increased with declining GFR [22].

Another important complication of CKD is infection of the respiratory and urinary tracts. Of the 1,498 inpatients with CRF in Chongqing, China, 71 suffered from tuberculosis, being atypical tuberculosis in more than half them. The prevalence of tuberculosis in the CRF inpatients $(4.74 \%)$ was significantly higher than that in residents (0.15\%), suggesting CRF patients are susceptible to tuberculosis. Renal function was inversely related to the prevalence of tuberculosis [23].

\section{Risk Factors of CKD in China}

IgAN is the most common CKD in China. IgAN is considered to be a multifactorial disease with genetic and environmental factors contributing to its pathogenesis. Megsin (SERPINB7) is an important candidate gene, which is predominantly expressed in glomerular mesangium and upregulated in IgAN [24]. Genetic study of 423 Chinese patients with biopsy-proven IgAN revealed that Megsin 2093C and 2180T alleles were significantly more 
often transmitted from heterozygous parents to patients than expected. The results suggest the genetic variation in Megsin confers susceptibility to IgAN in Chinese [25]. The study in 1,147 Chinese subjects revealed that there is no association between the VNTR polymorphism of the MUC20 gene and clinical manifestations in IgAN patients at the time of renal biopsy. However, IgAN patients with SL/LL genotypes had a higher risk of progression to ESRD [26]. The distribution of the uteroglobin G38A polymorphism (AA, AG and GG) showed no difference between Chinese patients with IgAN and normal controls, but patients with the 38AA genotype showed a higher odds ratio for decreased renal function compared to patients with the 38AG/GG genotype, suggesting the homogeneous 38AA genotype maybe one of the genetic markers for disease progression in Chinese patients with IgAN [27]. We investigated the relationship between angiotensin-converting enzyme (ACE) gene insertion/deletion polymorphisms and clinicopathological manifestations in Chinese patients with IgAN in 1997 and found higher frequencies for the DD genotype of the ACE gene in IgAN patients with hypertension and/or heavy proteinuria and/or severe glomerular sclerosis, suggesting the deletion polymorphism of the ACE gene may play a role, at least to some extent, in IgAN deterioration and progression [28].

Generally, it has been considered that impaired renal function, severe proteinuria, hypertension, glomerulosclerosis and interstitial fibrosis are the strongest and most reliable predictors of an unfavorable outcome in IgAN [29]. A retrospective study showed that proteinuria, elevated serum creatinine, glomerulosclerosis, crescent formation and interstitial fibrosis were independent risk factors in Chinese patients with IgAN. The patients with both renal insufficiency and proteinuria $>1.0 \mathrm{~g} / 24 \mathrm{~h}$ at the time of biopsy had a very poor 5-year renal survival rate [30]. Our study showed that severe glomerulosclerosis and tubulointerstitial lesions (TIL) were the primary pathologic characteristics of IgAN patients with renal dysfunction [31].

Hypertension is a common finding in progressive IgAN. We carried out a retrospective study in 540 Chinese patients with IgAN diagnosed by renal biopsy. The prevalence of hypertension in IgAN was $39.6 \%$ at the time of renal biopsy. Multivariate logistic regression analysis showed that factors independently influencing hypertension in patients with IgAN include age, familial history of hypertension, degree of proteinuria and hematuria, serum creatinine level, body weight and degree of renal arteriolar lesion [32]. Idiopathic IgAN is one of the main causes of secondary malignant hypertension, $1.2 \%$ of Chinese patients with IgAN presented malignant hypertension. Compared to primary malignant hypertension, proteinuria and hematuria were significantly higher, and glomerular injury was more severe in patients with malignant hypertension secondary to IgAN. The strict blood pressure control had a favorable effect on renal prognosis [33].

Most Chinese patients with IgAN had relatively severe lesions at the time of renal biopsy. We reviewed the clinical and pathological materials of 609 Chinese patients with IgAN: those with mild TIL accounted for $47.1 \%$, moderate TIL for $21.7 \%$, severe TIL for $16.6 \%$ and those without TIL for $14.6 \%$. Hypertension, the level of proteinuria, the scores of vascular lesions, total glomerular lesions, hypercellularity and glomerulosclerosis increased gradually with increasing TIL severity [34].

To clarify the characteristics of intrarenal arterial lesions in Chinese patients with IgAN and the main factors associated with them, we compared arterial lesions of 1,005 IgAN patients with those of 627 non-IgAN patients who had mesangial proliferating GN without IgA deposits and 221 patients with membranous nephropathy. The prevalence of intrarenal small artery and arteriolar lesions was $54.6 \%$ in IgAN patients, compared with 26.6 and $47.1 \%$ in non-IgAN and membranous nephropathy patients, respectively. The intrarenal arterial lesions in IgAN patients were associated with younger age, were more severe and exhibited a higher degree of hyaline changes compared with those in non-IgAN and membranous nephropathy. The severity of small arterial-arteriolar lesions was linked to several markers of disease progression, including hypertension, higher serum creatinine and uric acid, high urinary protein excretion, glomerulosclerosis, tubular atrophy and interstitial fibrosis [35].

\section{Prevention and Management of CKD in China}

Primary prevention of CKD is based on the management of obesity and its associated diseases such as type 2 diabetes as well as hypertension. Secondary prevention of CKD progression needs pharmacological approaches. Current management options for CKD are based on the control of known risk factors such as hypertension and proteinuria [16]. ACE inhibitors or angiotensin-2-receptor blockers have been widely used to prevent and treat CKD in China due to their antihypertensive and antiproteinuric effects [36]. Compared with placebo, benazepril 
(BZ) was associated with a $43 \%$ reduction in the risk of the primary end point (a doubling of the serum creatinine level, ESRD or death), a 52\% reduction in the level of proteinuria and a $23 \%$ reduction in the rate of decline in renal function in Chinese patients with serum creatinine levels of 3.1-5.0 mg/dl. These results suggest that BZ conferred substantial renal benefits in patients without diabetes who had advanced renal insufficiency [37]. Since the administration of ACE inhibitors or angiotensin receptor blockers may induce hyperkalemia and serum creatinine may rapidly increase in some patients with serum creatinine levels $>3 \mathrm{mg} / \mathrm{dl}$, close observation is mandatory during treatment.

IgAN is the most common GN in China. To evaluate the effects of therapy with urokinase (UK) and BZ or BZ alone on severe IgAN, 71 Chinese patients with IgAN, Lee's grade $\geq$ III and fibrinogen deposits were divided into two groups to be treated for 12 months with either $\mathrm{UK}+\mathrm{BZ}$ or BZ alone. Proteinuria was significantly decreased after 6 and 12 months of treatment in both groups compared with baseline, and the therapeutic efficiency of the UK-BZ combination was superior to that of $\mathrm{BZ}$ monotherapy. The endogenous creatinine clearance rate $\left(\mathrm{C}_{\mathrm{cr}}\right)$ was stable in the UK-BZ group, while $\mathrm{C}_{\mathrm{cr}}$ was significantly lower after 6 and 12 months in the BZ-alone group compared with baseline. The $\mathrm{C}_{\mathrm{cr}}$ of the two groups after 12 months of treatment were statistically different. These results suggest that combined therapy with $\mathrm{UK}$ and $\mathrm{BZ}$ was more effective than with $\mathrm{BZ}$ alone in reducing proteinuria and protecting renal function in Chinese patients with severe IgAN [38]. Additionally, we investigated the efficacy and safety of mycophenolate mofetil (MMF) in the treatment of severe IgAN. Sixty-two Chinese patients with biopsy-proven IgAN, pathologic grading IV-V by the Lee SMK system and urinary protein $>2.0 \mathrm{~g} /$ day were randomized to the MMF or prednisone group. After 3 months of treatment, a significant decrease in urinary protein and improvement in serum albumin were observed in the MMF group. After 18 months, the urinary protein of the MMF group was significantly lower than that of the prednisone group. Repeated renal biopsy showed that interstitial cell infiltration was alleviated after the treatment in both groups. The results demonstrated that MMF was effective in lowering proteinuria in Chinese patients with severe IgAN [39]. However, there are reports that MMF was ineffective in IgAN [40]. To evaluate the effect and safety of the Chinese medicine compound Shenhua Tablet on IgAN patients with traditional Chinese medicine Qi-Yin deficiency syndrome, a prospective, multicenter, double-blind, double-dummy, randomized, controlled trial was conducted. After 12 weeks of treatment, Shenhua Tablet, just like the positive control medicine fosinopril, effectively decreased proteinuria and improved IgAN without serious adverse reactions [41].

$\mathrm{LN}$ is the most common form of secondary renal disease diagnosed by renal biopsy in China. Mok et al. [42] examined the long-term outcome of 183 Southern Chinese patients with LN. The mean duration of follow-up from the renal biopsy was $130.7 \pm 5.9$ months (range, 13-260 months). The overall 5-, 10- and 15-year survival and renal survival (survival without dialysis) rates were 98.9, 94.4 and $94.4 \%$ and $92.1,81.2$ and $75.2 \%$, respectively. Multivariate analysis showed persistent hypertension, class IV nephritis and incomplete renal remission in the 1st year as independent risk factors for renal failure. These results showed that the renal survival rate of the LN patients from South China was similar to that of most reported series of white patients [42]. Severe edema and lower serum albumin were associated with mortality in LN patients. Male sex, postponing hospital admission after onset of renal failure, atrophic renal size and high concentrations of serum phosphate were all predictors of poor recovery of renal function. Intravenous high-dose cyclophosphamide therapy had a protective effect on the recovery of renal function [43]. MMF has been widely used in Chinese patients with proliferative LN. Seventyfive patients with biopsy-proven active diffuse proliferative LN from nine hospitals in China were treated with a combined regimen of MMF and steroids. The hemoglobin level increased, proteinuria decreased, the proportion of patients with positive anti-dsDNA antibody and hypocomplementemia was significantly reduced after 6 months of MMF treatment. Repeat renal biopsy in 38 patients 3-6 months after the treatment showed a significant decrement in the renal pathological activity index. These results suggest that MMF combined with steroids may provide an effective therapy for diffuse proliferative LN [44]. Another Chinese prospective, multicenter, controlled clinical trial showed that leflunomide was effective as induction therapy for proliferative LN. There was no statistical difference between leflunomide-treated patients and cyclophosphamide-treated patients [45].

\section{CKD: A Great Challenge to Chinese Nephrologists}

The number of newly diagnosed CKD cases is increasing, and most of them were diagnosed with elevated serum creatinine in China. The percentage of CKD patients 
who achieved their treatment goal was low, and few patients started renal replacement therapy in time. Apart from economic problems, delayed diagnosis and unwillingness of patients to accept the fact remained the main reason for delayed renal replacement therapy. These results indicate that Chinese nephrologists should provide health education and consultation for CKD patients to improve their compliance [46].

Chinese nephrologists have been acting for CKD patients. On March 9, 2006 (the first 'World Kidney Day') [47], the CSN carried out a lot of activities at Beijing and other areas and declared the 'World Kidney Day' proposal, appealing to the whole public to be aware of CKD and its associated diseases [48]. On March 8, 2007 (the second 'World Kidney Day'), activities have been per- formed throughout China. The message of the World Kidney Day is that kidney disease is common and harmful, but treatable [49]. The CSN will launch a study on the CKD epidemic in China, in order to facilitate early prevention of CKD by controlling diabetes, hypertension and hyperlipidemia, and furthermore to have patients with mild or moderate CKD treated as early as possible.

\section{Acknowledgements}

This study was supported by grants from the Creative Research Group Fund (30121005), General Program (30570856), Key Program (30630033) of the National Natural Science Foundation of China and the National Basic Research Program of China (2006CB507400).

\section{References}

-1 Levey AS, Coresh J, Balk E, Kausz AT, Levin A, Steffes MW, Hogg RJ, Perrone RD, Lau J, Eknoyan G; National Kidney Foundation: National Kidney Foundation practice guidelines for chronic kidney disease: evaluation, classification, and stratification. Ann Intern Med 2003;139:137-147.

$\checkmark 2$ National Kidney Foundation: K/DOQI clinical practice guidelines for chronic kidney disease: evaluation, classification, and stratification. Am J Kidney Dis 2002;39:S1S246.

3 Keane WF, Eknoyan G: Proteinuria, albuminuria, risk, assessment, detection, elimination (PARADE): a position paper of the National Kidney Foundation. Am J Kidney Dis 1999;33:1004-1010.

4 Coresh J, Astor BC, McQuillan G, Kusek J, Greene T, Van Lente F, Levey AS: Calibration and random variation of the serum creatinine assay as critical elements of using equations to estimate glomerular filtration rate. Am J Kidney Dis 2002;39:920-929.

$\checkmark 5$ Zuo L, Ma YC, Zhou YH, Wang M, Xu GB, Wang HY: Application of GFR-estimating equations in Chinese patients with chronic kidney disease. Am J Kidney Dis 2005;45: 463-472.

6 Ma YC, Zuo L, Chen JH, Luo Q, Yu XQ, Li Y, Xu JS, Huang SM, Wang LN, Huang W, Wang M, Xu GB, Wang HY: Modified glomerular filtration rate estimating equation for Chinese patients with chronic kidney disease. J Am Soc Nephrol 2006;17:29372944.

7 Coresh J, Astor BC, Greene T, Eknoyan G, Levey AS: Prevalence of chronic kidney disease and decreased kidney function in the adult US population: Third National Health and Nutrition Examination Survey. Am J Kidney Dis 2003;41:1-12.
-8 Chadban SJ, Briganti EM, Kerr PG, Dunstan DW, Welborn TA, Zimmet PZ, Atkins RC: Prevalence of kidney damage in Australian adults: the AusDiab kidney study. J Am Soc Nephrol 2003; 14:S131-S138.

9 Chen J, Wildman RP, Gu D, Kusek JW, Spruill M, Reynolds K, Liu D, Hamm LL, Whelton PK, He J: Prevalence of decreased kidney function in Chinese adults aged 35 to 74 years. Kidney Int 2005;68:2837-2845.

10 Zhang L, Zuo L, Xu G, Wang F, Wang M, Wang S, Lv J, Liu L, Wang H: Communitybased screening for chronic kidney disease among populations older than 40 years in Beijing. Nephrol Dial Transplant 2007;22: 1093-1099.

11 Chen W, Wang H, Dong XQ, He HJ, Liu QH, Luo N, Tan JQ, Lin JX, Nie J, Mao HP, Chen WQ, Yu XQ: Epidemiologic study of chronic kidney disease in Guangzhou urban area (in Chinese). Chin J Nephrol 2007;23:147-151.

12 Guo LZ, Zhang LX, Wang XG, Zhang P, Zhao QP, Wang F, Wang HY: Screening for chronic kidney disease in a village of Zhejiang province (in Chinese). Chin J Nephrol 2007;23:152-156.

13 Li LS, Liu ZH: Epidemiologic data of renal diseases from a single unit in China: analysis based on 13,519 renal biopsies. Kidney Int 2004;66:920-923.

14 Liu G, Ma X, Zou W: The prevalence of biopsy-proven renal disease in adults between two periods over the past 10 years (in Chinese). J Clin Intern Med 2004;21:834-838.

15 Lysaght MJ: Maintenance dialysis population dynamics: current trends and long-term implications. J Am Soc Nephrol 2002;13:3740.

16 Meguid El Nahas A, Bello AK: Chronic kidney disease: the global challenge. Lancet 2005;365:331-340.
17 United States Renal Data System: Annual data report: incidence and prevalence of ESRD (2003). Am J Kidney Dis 2003;42:S37S173.

18 Dialysis and Transplantation Registration Group, Chinese Society of Nephrology: The report about the registration of dialysis and transplantation in China 1999 (in Chinese). Chin J Nephrol 2001;17:77-78.

19 Shanghai Dialysis and Transplantation Registration Group: The report about the registration of dialysis and transplantation in Shanghai 1999 (in Chinese). Chin J Nephrol 2001;17:83-85.

20 Zhang L, Wang M, Wang H: Acute renal failure in chronic kidney disease - clinical and pathological analysis of 104 cases. Clin Nephrol 2005;63:346-350.

21 Zhang L, Zuo L, Wang F, Wang M, Wang S, Lv J, Liu L, Wang H: Cardiovascular disease in early stages of chronic kidney disease in a Chinese population. J Am Soc Nephrol 2006; 17:2617-2621.

22 Hou FF, Ma ZG, Mei CL, Rong S, Huang SM, Liu XR, Yuan WJ, Guo YS, Wang L, He Q, Wang XL, Sang XH, Li XL: Cardiovascular disease in Chinese chronic renal insufficiency patients - epidemiology survey (in Chinese). Zhonghua Yi Xue Za Zhi 2005;85:458463.

23 Yuan FH, Guang LX, Zhao SJ: Clinical comparisons of 1,498 chronic renal failure patients with and without tuberculosis. Ren Fail 2005;27:149-153.

24 Miyata T, Nangaku M, Suzuki D, Inagi R, Uragami K, Sakai H, Okubo K, Kurokawa K: A mesangium-predominant gene, megsin, is a new serpin upregulated in IgA nephropathy. J Clin Invest 1998;102:828-836. 
25 Li YJ, Du Y, Li CX, Guo H, Leung JC, Lam MF, Yang N, Huang F, Chen Y, Fang JQ, Maxwell PH, Lai KN, Wang Y: Family-based association study showing that immunoglobulin A nephropathy is associated with the polymorphisms $2093 \mathrm{C}$ and $2180 \mathrm{~T}$ in the 3' untranslated region of the Megsin gene. J Am Soc Nephrol 2004;15:1739-1743.

-26 Li G, Zhang H, Lv J, Hou P, Wang H: Tandem repeats polymorphism of MUC20 is an independent factor for the progression of immunoglobulin A nephropathy. Am J Nephrol 2006;26:43-49.

-27 Lu JC, Zhang H, Chen YQ, Liu G, Wang HY: Uteroglobin G38A polymorphism is associated with the progression of IgA nephropathy in Chinese patients (in Chinese). Zhonghua Nei Ke Za Zhi 2004;43:37-40.

28 Chen X, Liu S, Ye Y, Xu Q: Association of angiotensin-converting enzyme gene insertion/deletion polymorphism with the clinico-pathological manifestations in immunoglobulin A nephropathy patients. Chin Med J (Engl) 1997;110:526-529.

29 D'Amico G: Natural history of idiopathic IgA nephropathy: role of clinical and histological prognostic factors. Am J Kidney Dis 2000;36:227-237.

-30 Yang NS, Wu QQ, Du Y, Zhang R, Li YJ, Ye RG, Yu XQ: Risk factors affecting the longtime outcome of IgA nephropathy (in Chinese). Zhonghua Nei Ke Za Zhi 2005;44:597600.

-31 Chen X, Wang Y, Tang L, Liu S, Wu J: Clinical evaluation of renal biopsy in 106 patients of IgA nephropathy with chronic renal failure (in Chinese). Chin J Nephrol 2003;19:15-19.

32 Zhuang Y, Chen X, Zhang Y: Multivariate analysis of influencing factors for hypertension in patients with IgA nephropathy (in Chinese). Zhonghua Nei Ke Za Zhi 2000;39: 371-375.
33 Chen Y, Tang Z, Yang G, Shen S, Yu Y, Zeng C, Chen H, Liu ZH, Li LS: Malignant hypertension in patients with idiopathic IgA nephropathy. Kidney Blood Press Res 2005;28: 251-258.

34 Zhang Y, Chen X, Zhuang Y: Clinical analysis of tubulointerstitial lesions in IgA nephropathy (in Chinese). Zhonghua Nei Ke Za Zhi 2001;40:613-617.

35 Wu J, Chen X, Xie Y, Yamanaka N, Shi S, Wu D, Liu S, Cai G: Characteristics and risk factors of intrarenal arterial lesions in patients with IgA nephropathy. Nephrol Dial Transplant 2005;20:719-727.

36 Shi X, Chen X, Liu S, Zhuang Y, Zhang Y: The effects of angiotensin-converting enzyme inhibitor on IgA nephropathy and the influencing factors (in Chinese). Zhonghua Nei Ke Za Zhi 2002;41:399-403.

37 Hou FF, Zhang X, Zhang GH, Xie D, Chen PY, Zhang WR, Jiang JP, Liang M, Wang GB, Liu ZR, Geng RW: Efficacy and safety of benazepril for advanced chronic renal insufficiency. N Engl J Med 2006;354:131-140.

38 Chen X, Qiu Q, Tang L, Liu S, Cai G, Liu H, Xie Y: Effects of co-administration of urokinase and benazepril on severe IgA nephropathy. Nephrol Dial Transplant 2004;19:852857.

39 Chen X, Chen P, Cai G, Wu J, Cui Y, Zhang Y, Liu S, Tang L: A randomized control trial of mycophenolate mofetil treatment in severe IgA nephropathy (in Chinese). Zhonghua Yi Xue Za Zhi 2002;82:796-801.

40 Maes BD, Oyen R, Claes K, Evenepoel P, Kuypers D, Vanwalleghem J, Van Damme B, Vanrenterghem YF: Mycophenolate mofetil in IgA nephropathy: results of a 3-year prospective placebo-controlled randomized study. Kidney Int 2004;65:1842-1849.

41 Chen XM, Chen YP, Chen J: Multicentered, randomized, controlled clinical trial on patients with IgA nephropathy of Qi-yin deficiency syndrome type (in Chinese). Zhongguo Zhong Xi Yi Jie He Za Zhi 2007;27: 101-105.
42 Mok CC, Wong RW, Lau CS: Lupus nephritis in Southern Chinese patients: clinicopathologic findings and long-term outcome. Am J Kidney Dis 1999;34:315-323.

43 Liang L, Yang X, Xu H, Zhan Z, Ye Y, Yu X, Chen W: Clinical predictors of recovery and complications in the management of recentonset renal failure in lupus nephritis: a Chinese experience. J Rheumatol 2004;31:701706.

44 Li L, Wang H, Lin S, Chen X, Chen Y, XU J, Chen N, Li X, Qian J, Hu W: Mycophenolate mofetil treatment for diffuse proliferative lupus nephritis: a multicenter clinical trial in China (in Chinese). Zhonghua Nei Ke Za Zhi 2002;41:476-479.

45 Cui TG, Hou FF, Ni ZH, Chen XM, Zhang FS, Zhu TY, Zhao XZ, Bao CD, Zhao $\mathrm{MH}$ Wang GB, Qian JQ, Cai GY, Li YN, Lu FM, Mei CL, Zou WZ, Wang HY: Treatment of proliferative lupus nephritis with leflunomide and steroid: a prospective multi-center controlled clinical trial (in Chinese). Zhonghua Nei Ke Za Zhi 2005;44:672-676.

46 Zou L, Wang M, Wang H: Habit of nephrologists in monitoring and treatment of chronic kidney disease in China (in Chinese). Chin J Nephrol 2005;21:127-132.

47 Collins AJ, Couser WG, Dirks JH, Kopple JD, Reiser T, Riella MC, Robinson S, Shah SV Wilson A: World Kidney Day: an idea whose time has come. Kidney Int 2006;69:781782.

48 Chen X, Zhang Y: Propagandize world kidney day actively, appeal to the society for the attention to chronic kidney disease (in Chinese). Chin J Nephrol 2006;22:65-66.

49 Levey AS, Andreoli SP, Dubose T, Provenzano R, Collins AJ: Chronic kidney disease: common, harmful and treatable - World Kidney Day 2007. Am J Nephrol 2007;27: 108-112. 